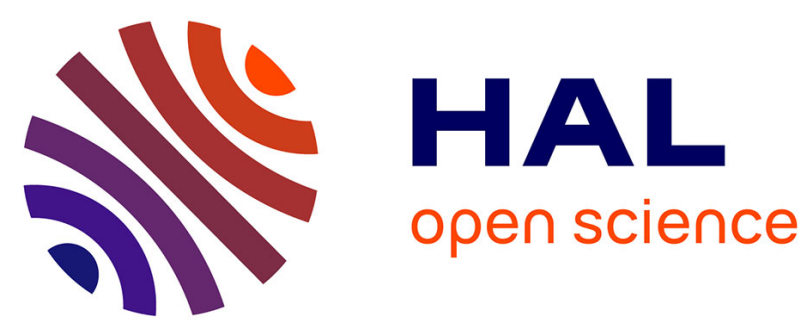

\title{
Salt exclusion in charged porous media: a coarse-graining strategy in the case of montmorillonite clays
}

Marie Jardat, Jean-François Dufrêche, Virginie Marry, Benjamin Rotenberg, Pierre Turq

\section{To cite this version:}

Marie Jardat, Jean-François Dufrêche, Virginie Marry, Benjamin Rotenberg, Pierre Turq. Salt exclusion in charged porous media: a coarse-graining strategy in the case of montmorillonite clays. Physical Chemistry Chemical Physics, 2009, 11 (12), 10.1039/B818055E . hal-01917646

\section{HAL Id: hal-01917646 \\ https://hal.sorbonne-universite.fr/hal-01917646}

Submitted on 9 Nov 2018

HAL is a multi-disciplinary open access archive for the deposit and dissemination of scientific research documents, whether they are published or not. The documents may come from teaching and research institutions in France or abroad, or from public or private research centers.
L'archive ouverte pluridisciplinaire HAL, est destinée au dépôt et à la diffusion de documents scientifiques de niveau recherche, publiés ou non, émanant des établissements d'enseignement et de recherche français ou étrangers, des laboratoires publics ou privés. 


\title{
Salt exclusion in charged porous media: A coarse-graining strategy in the case of montmorillonite clays
}

\author{
Marie Jardat*, Jean-François Dufrêche ${ }^{\dagger}$, Virginie Marry, Benjamin Rotenberg and Pierre Turq \\ UPMC Univ Paris 06, UMR 7612, Laboratoire Liquides Ioniques et Interfaces Chargées, F-75005, Paris, \\ CNRS, UMR 7612, Laboratoire LI2C, 4 place Jussieu, F-75005 Paris, France
}

\begin{abstract}
We study the exclusion of salt from charged porous media (Donnan effect), by using a coarsegrained approach. The porous medium is a lamellar system, namely a Montmorillonite clay, in contact with a reservoir which contains an electrolyte solution. We develop a specific coarse-graining strategy to investigate the structural properties of this system. Molecular simulations are used to calibrate a mesoscopic model of the clay micropore in equilibrium with a reservoir. Brownian Dynamics simulations are then used to predict the structure of ions in the pore and the amount of $\mathrm{NaCl}$ salt entering the pore as a function of the pore size (the distance $L$ between clay surfaces) and of the electrolyte concentration in the reservoir. These results are also compared to the predictions of a Density Functional Theory which takes into account the excluded volumes of ions. We show that the calibration of the mesoscopic model is a key point and has a strong influence on the result. We observe that the salt exclusion increases when $\kappa L$ decreases (where $\kappa$ is the inverse of the Debye length) and that this effect is modulated by the correlations between ions. Two different regimes are revealed. At low concentration in the reservoir, we observe a regime controlled by electrostatics: the Coulomb attraction between ions increases the amount of salt in the interlayer space. On the opposite, at high concentration in the reservoir, the excluded volume effect dominates.
\end{abstract}

\section{INTRODUCTION}

A common feature of most materials acting as membranes or retention barriers in various industrial and environmental processes is to consist of charged porous materials. Examples ranging from polymeric or inorganic membranes for ionic exchange or reverse osmosis (e.g. for seawater desalinization) to natural minerals in soils (through which rainwater flows) and deeper geological formations (e.g. aquifers, or cap rocks above oil and gas reservoirs) illustrate the diversity of situations in which the flow of a fluid through a charged porous medium plays a crucial role. In particular, clays are considered as suitable materials for the confinement of toxic or radioactive waste $[1,2]$. In the very long term, the geological medium is expected to act as the main barrier preventing the release of waste in the biosphere. This is one of the reason explaining why the transport of charged species through clays has recently received a growing attention, both from the experimental and theoretical points of view. Owing to the very low permeability of compacted clay, the diffusion of ions, as tracers or in finite concentrations, is thought to be the main transport mechanism.

The mechanical (swelling) and retention properties of clays are related to their permanent negative charge, compensated by counterions. They consist of aluminosilicate layers stacked into anisotropic particles. Most of the counterions are located in the nanoporosity left between the mineral layers. The imperfect packing of these particles into platelets gives rise to larger pores, with a size distribution that depends on the precise nature of

\footnotetext{
*marie.jardat@upmc.fr

$\dagger$ jean-francois.dufreche@upmc.fr
}

the clay and the sample history (formation, compaction, hydration state,...). When placed in contact with a water or electrolyte reservoir, these micropores (ranging from a few $\mathrm{nm}$ to tens of $\mathrm{nm}$ ) can be saturated with water and some salt will also be allowed into this porous material. A schematic representation of this system is drawn in Figure 1.

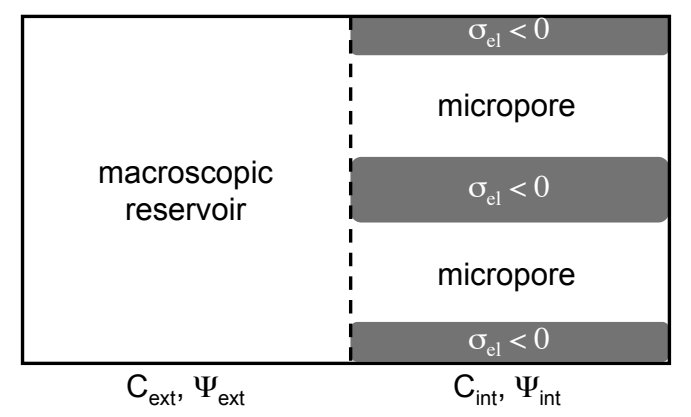

FIG. 1: Schematic representation of the system studied in this paper: A lamellar charged porous medium is in contact with a reservoir. The surface charge is compensated by counterions. Because of this excess charge, the electrostatic potential in the micropore $\Psi_{\text {int }}$ differs from that in the reservoir $\Psi_{\text {ext }}$. The reservoir contains a bulk electrolyte aqueous solution at a concentration $C_{\text {ext }}$, which partly penetrates into the micropores. We study how the equilibrium concentration $C_{\text {int }}$ inside the micropore depends on the value of $C_{\text {ext }}$ (Donnan effect).

The diffusion of ions through this porous medium is driven by the ion distributions in the micropores. For example, anions are repelled by the negative charge of the clay layers and are excluded from the smallest pores of the material, which effectively acts as a barrier for these ions. This simple fact partly explains the experimental observation that anions diffuse slower than water 
and cations through clay barriers. However, a clear explanation of this phenomenon is still lacking. In particular, little is known about how the size of the micropore affects the amount of salt (including the anions) that can be incorporated in the medium as a function of the salt concentration in the reservoir. The inclusion of salt plays a crucial role in screening the repulsive electrostatic interactions between clay particles, and thus in the mechanical stability of the material. More directly, a larger salt concentration increases the amount of potentially hazardous species passing through it.

The partial insertion of the electrolyte solution into the micropores is an example of the so-called Donnan effect, as it is represented in Fig. 1. The Donnan effect usually describes the behavior of charged particles near a semi-permeable membrane, which fail to distribute evenly across the two sides of the membrane, because of the presence of a charged substance unable to pass through it. This creates an electrostatic potential barrier between the two compartments. In the present case, the difference in electrostatic potential between the two "compartments", namely the reservoir and the micropore, is due to the immobile charged clay layers. The Donnan equilibrium, which appears between the ionic concentration in the micropores and in the reservoir, strongly depends on the porosity of the system, represented in the simple model of Fig. 1 by the intersurface distance, denoted by $L$ in the following. Typically, if $L$ is much bigger than the Debye length of the external electrolyte solution, the charge of the porous material is screened by the counterions and the coions (anions, since clays are negatively charged) can penetrate into the pore. On the other hand, if $L$ is much smaller than the Debye length, a Donnan electrostatic potential difference arises between the fluid inside the micropore and the reservoir [3]. The anions are then excluded from the pores. The Donnan effect can be quantified by calculating the concentration in the micropores of anions, namely coions of the porous medium, as a function of their concentration in the reservoir, for a given porosity $L$.

The standard description of the Donnan effect is based on continuous models in which ions are treated as point particles interacting at the mean-field level via the electrostatic potential. Such an approach, commonly based on the Poisson-Boltzmann equation $(\mathrm{PB})$, is valid when the size of the ions is small compared to the distance between them and to the size of the pores. Some recent works based on continuous models used atomic simulations to improve the description of interactions between ions and the walls representing the porous medium [4-7]. For nanometric pores and high salt concentrations that might be encountered under geological conditions, adopting continuous descriptions requires great caution. At the other extreme, molecular simulations have proved very powerful for the study of clay interlayers (the nanoporosity containing a few discrete layers of water inside the clay particles), and more recently on the direct vicinity of basal and lateral surfaces of clay particles. They pro- vided new insight in experimental observations on the structural [8-13], thermodynamical [14-20] and dynamical [21-27] properties of clays. Most notably, they revealed at the molecular scale the subtle interplay governing the interactions between the mineral layers, the counterions and water molecules. This specificity was also observed on external surfaces of clay particles [28, 29], and could influence their effective charge, modifying in turn the properties further away from the surface.

The atomistic simulation of larger, water saturated pores, remains however computationally expensive [30]. Increasing the system size indeed implies a larger number of interactions between atoms to be computed and a longer (not only computational, but also physical) time needed to sample correctly the larger phase space. The objective of this paper is thus twofold: (i) we aim at studying the Donnan effect in a lamellar charged porous medium, and (ii) to attain this goal, we develop a coarsegraining procedure. More precisely, molecular simulations are first used to calibrate a mesoscopic model of a clay micropore in equilibrium with a reservoir of an aqueous sodium chloride solution. Brownian simulations are then used to study the Donnan effect, i.e. to evaluate the amount of salt entering into the pore as a function of pore size and salt concentration in the reservoir. These results are finally compared to the predictions of Density Functional Theory. The continuity between the levels of description allows us to assess the validity of coarser models, which are simpler and faster to make predictions, e.g. in an engineering context.

The paper is organized as follows. In Sec. II A, the description of the clay system at the atomic scale allows us to draw the principles of our coarse-graining procedure. The different theoretical methods used to develop the coarse-grained model and to study the Donnan effect are then described in Sec. IIB-IID. Sec. III A is devoted to the calibration of coarse-grained interaction parameters. In Sec. III B, the insertion properties of co-ions in the porous medium as functions of the interlayer spacing and of the electrolyte concentration in the reservoir are investigated for the coarse-grained model. Results obtained from numerical simulations and DFT are compared and discussed. The last section contains a conclusive discussion.

\section{SYSTEM AND METHODS}

\section{A. System and coarse-graining procedure}

The clay under study is a Montmorillonite, whose properties have been previously successfully investigated from atomistic simulations. At the atomic level, each clay layer consists of one sheet of octahedral aluminum oxide between two sheets of tetrahedral silicon oxides. A fraction of $\mathrm{Al}^{3+}$ and $\mathrm{Si}^{4+}$ are substituted by cations of lower valence, $\mathrm{Mg}^{2+}$ and $\mathrm{Al}^{3+}$ respectively. The Montmorillonite studied here contains only 

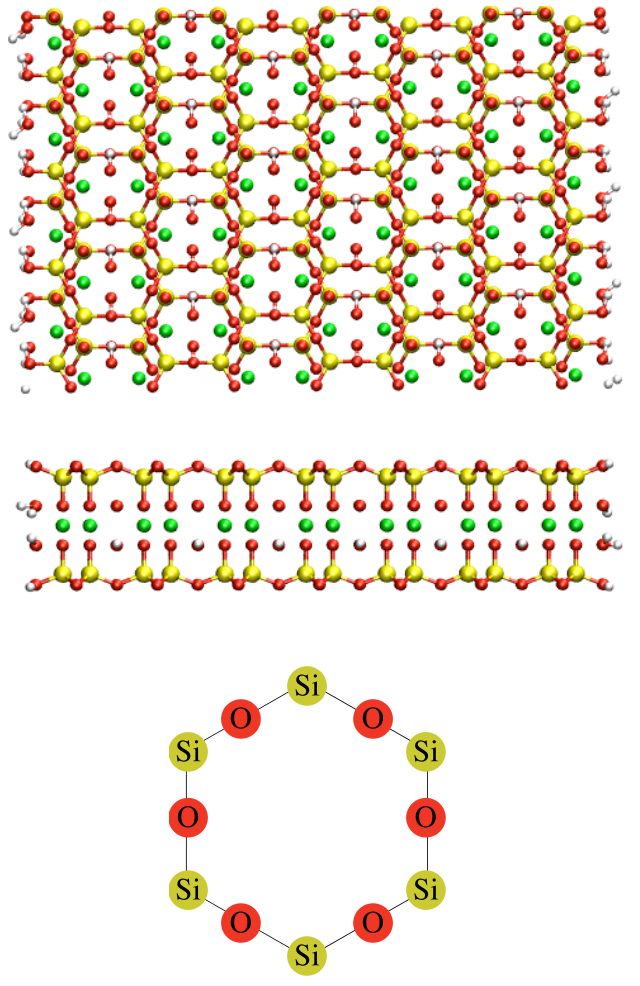

FIG. 2: Atomic structure of the clay layer. Top: Surface of the clay layer; Middle: Lateral view of the clay, whose thickness is equal to $6.54 \AA$; Bottom: Disposition of silicon and oxygen atoms over the surface of the clay. The $\mathrm{O}$ atoms are in red, $\mathrm{H}$ in white, $\mathrm{Si}$ in yellow, $\mathrm{Al}$ and $\mathrm{Mg}$ in green.

octahedral substitutions and its unit cell formula is: $\mathrm{Na}_{0.75} \mathrm{Si}_{8}\left(\mathrm{Al}_{3.25} \mathrm{Mg}_{0.75}\right) \mathrm{O}_{20}(\mathrm{OH})_{4}$. The negative charge is thus $0.75 e$ per unit cell $\left(\sigma=-0.016 e / \AA^{2}\right.$. It is located in the middle of the clay layer and is compensated by 0.75 monovalent counterions $\mathrm{Na}^{+}$. The atomic structure of the clay layer was taken from X-ray diffraction measurements [31, 32]. The layer thickness is $6.54 \AA$. This atomic structure is given in Fig. 2; the honeycomb disposition of silicon and oxygen atoms near the surface of the sheet is also indicated.

In the present study, these hydrated clay layers are assumed to be in contact with a reservoir which consists of an aqueous electrolyte solution. As already mentioned in the introduction, the atomistic simulation of such a system for several interlayer spacings and several electrolyte concentrations in the reservoir is too expensive. Thus, we propose a coarse-graining approach which relies on two main simplifications: $(i)$ the solvent is assumed to be a continuous medium, characterized by its dielectric constant $\varepsilon_{r}$ and ( $\left.i i\right)$ the clay layer is described by an assembly of neutral and charged spheres, with the same charge density as the atomic one, as shown in Fig. 3. The first assumption is appealing because continuous solvent models have proved since a long time to describe accurately structural and dynamical properties of aqueous so-
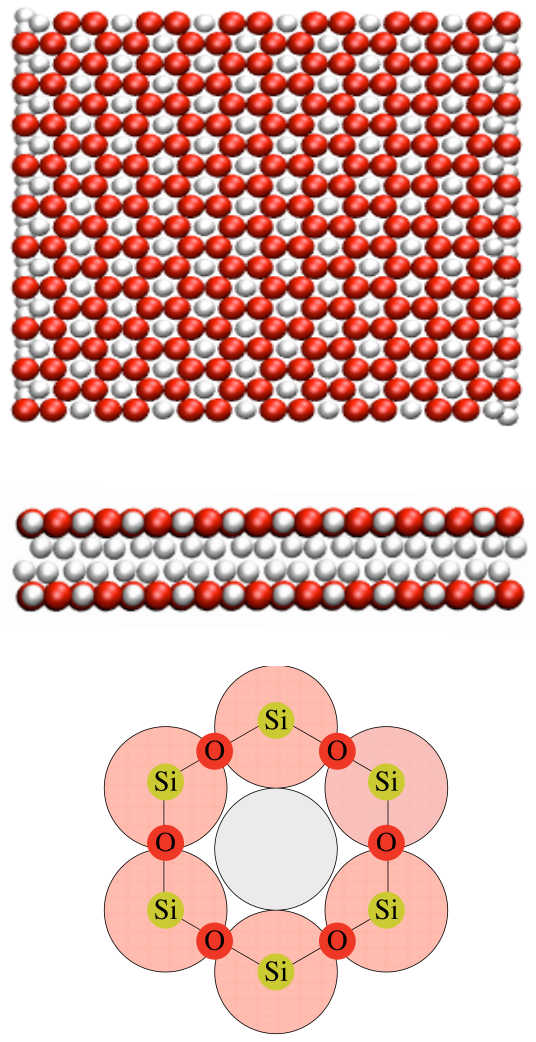

FIG. 3: Coarse-grained description of the clay layer. Top: Surface of the clay ; Middle: Lateral view of the clay, whose thickness is equal to $6.54 \AA$; Bottom: Disposition of charged and neutral spheres used to account for the charge disposition and charge density near the surface of the clay (positions of silicon and oxygen atoms over the surface of the atomic clay is also indicated). The charged spheres are in red, neutral spheres in white. The clay surface is a honeycomb of such hexagones.

lutions of simple electrolytes [33, 34]. Usually, either the so-called primitive model of electrolytes which treats the ions as charged hard spheres or its soft-core variant are used $[35,36]$. Here, ions interact through the Coulomb potential and a $1 / r^{12}$ short-range repulsion. The second assumption ( $i i)$ is necessary to keep the essential features of clay layers while decreasing the number of particles of the system.

The disposition of charged and neutral spheres which constitute the clay layer at the mesoscopic level mimic the atomic disposition of charged sites. The positions of charged and neutral spheres follow a honeycomb hexagonal geometry, with a distance between the sphere centers of $1.3 \AA$ (see Fig. 3), which corresponds to the distance between silicon atoms at the atomic scale. The charged spheres (in red in Fig. 3) represent the net charge of the clay surface mainly located over the silicon atoms. A neutral sphere is added in between the charged ones to account for the non-penetrability of the clay layer. The charged spheres of the coarse-grained clay surface have a 
charge equal to $-0.0625 \mathrm{e}$, which yields exactly the same charge density as in the atomic layer. The main difference between the atomic and the coarse-grained clay layers comes from the fact that the charges are located on the surface of the clay in the coarse-grained description. In the atomic description, $\mathrm{Al}, \mathrm{Mg}, \mathrm{Si}$ and $\mathrm{H}$ atoms are positively charged, whereas $\mathrm{O}$ atoms of the surface and of the tetrahedric and octahedric sheets are negatively charged. Nevertheless, as it is demonstrated below, the coarse-grained model results in ionic distributions in the direction perpendicular to the clay surface in good agreement with atomic ones. Two layers of charged and neutral spheres are used to describe the two basal surfaces of one clay layer. The layer thickness is the same as the atomic one and edge neutral spheres are added to prevent ions from prenetrating inside the clay (see Fig. 3, middle).

As for the modelling of ions of the electrolyte solutions, every ion is assumed to interact with the clay surfaces through a short-range repulsion (proportional to $\left.1 / r^{12}\right)$ in addition to the Coulomb potential. For the sake of simplicity, this short-range repulsion between ions and the spheres which constitute the clay is assumed to be the same for neutral and charged spheres. The key point of our coarse-graining procedure is that the range of this ion/surface repulsion is calibrated by using Molecular Dynamics simulations. More precisely, the distribution profiles of ions in the direction perpendicular to the clay surface are computed both from Molecular Dynamics simulations and from Brownian Dynamics simulations on the mesoscopic level, for several ion/clay repulsion parameters. These calculations are performed for systems containing infinite clay layers (no reservoir) with a large interlayer distance and only for one ionic concentration. The fitted parameters are then kept for all other calculations. The details of this fitting procedure are given in section III A.

On the mesoscopic scale, alternative theoretical descriptions of the coarse-grained system may also be used to study the Donnan effect. The simplest model is to treat ions as point charges and the clay surfaces as infinite and uniformly charged planes in contact with a reservoir. Thus, the concentration of coions between the charged planes can be obtained in the Poisson-Boltzmann approximation. This description is again a coarse-grained description of the real system, in which the excluded volume of the clay (namely the minimal distance of approach between ions and the clay) has to be calibrated using results at a more detailed scale. Nevertheless, such a coarsegrained description, which assumes that ions are point charges, neglects the excluded volumes of ions themselves so that it is unable to account for the insertion of ions for relatively small interlayer distances or for large external electrolyte concentration. Thus, we propose a more precise theoretical description of the system, which takes into account the excluded volumes of ions. In the present paper, Density Functional Theory (DFT) is used to calculate the ionic densities in the porous medium in contact with a reservoir by using the Mean Spherical Approximation (MSA) to account for the excluded volumes of ions. Since the Poisson-Boltzmann aproximation can be rephrased as a DFT calculation, we keep the DFT formalism in both cases, when ions are assumed to be either point charges or charged hard spheres. The principles of this calculation are given in Sec. IID.

\section{B. Molecular Dynamics simulations}

Molecular dynamics simulations are used here only to perform the coarse-graining of the interaction between ions and the clay surfaces. The simulation box at the atomic level contains one clay layer of $6.54 \AA$ width along the $z$-direction, cut into two halves, whose horizontal dimensions are $35.88 \times 41.44 \AA^{2}$. The net charge of the clay surface is then $-24 e$. The interlayer distance $L$ is here the distance between the oxygen atoms of the surfaces of the clay. Periodic boundary conditions in all dimensions are used so that a periodic stacking of infinite clay layers is simulated. The space between the layers is filled with water, $24 \mathrm{Na}^{+}$counterions and a salt at the concentration $C_{\text {salt }}$. We use the SPC/E water model [37], known to accurately reproduce structural and dynamical properties of water, in particular its dielectric constant and diffusion coefficient. The force field used to describe the interactions between clay atoms, water atoms and ions consists of pairwise interactions. The interaction between two atoms contains a direct electrostatic (Coulomb) contribution and a Lennard-Jones potential to account for short-range repulsion and long-range dispersion forces:

$$
V_{i j}\left(r_{i j}\right)=\frac{q_{i} q_{j}}{4 \pi \epsilon_{0} r_{i j}}+4 \epsilon_{i j}\left[\left(\frac{\sigma_{i j}}{r_{i j}}\right)^{12}-\left(\frac{\sigma_{i j}}{r_{i j}}\right)^{6}\right]
$$

where $q_{i}$ and $q_{j}$ are the charges of atoms $i$ and $j$, and $r_{i j}$ the distance between them. The Lennard-Jones parameters $\sigma_{i j}$ and $\epsilon_{i j}$ are computed from individual $\sigma_{i i}$ and $\epsilon_{i i}$ using Lorentz-Berthelot mixing rules. We use the individual parameters for ions in SPC/E water of Dang et al. which were shown to correctly describe the dynamics of ions in bulk water [38]; the parameters for clay atoms are the ones of Smith [11]. The values of all these parameters are exactly the same as those given in ref. [39]. This description of the clay/water interface has already proved to describe reasonably well a number of experimental results on the thermodynamical, structural and dynamical properties of bulk clays at low hydration (interlayer) [22, 23, 26, 27]. The long-range electrostatic interactions are computed via three dimensional Ewald summation [40]. Molecular dynamics simulations are performed in the canonical $(N, V, T)$ ensemble using the DLPOLY simulation package. 


\section{Brownian Dynamics simulations}

As stated before, the confined solution under study is a complex multicomponent system of clay, solvent, cations and anions (the counterions and coions). The first coarse-graining step is to replace the solvent (water) by its effective effects on the ions, thus treating it as a continuous medium (McMillan-Mayer level of description). In the framework of the continuous solvent model, Brownian Dynamics (BD) is a simulation method allowing one to compute the trajectories of solute particles. It is able to take into account the direct interaction between solute particles as well as the indirect hydrodynamic interactions. In the present study, we are interested in the structural properties of the system so that hydrodynamic interactions can be neglected. Here, the interest of Brownian Dynamics is mainly that it allows one to sample the phase space more efficiently than Monte Carlo simulations.

On the mesoscopic time scale of Brownian Dynamics, the motions are described in the position space only and are governed by the generalized Smoluchowski equation. When hydrodynamic interactions are neglected, the corresponding stochastic equation for the displacement of $N$ particles included in the simulation box from time $t$ to time $t+\delta t$ is $[41]$

$$
\mathbf{r}(t+\delta t)=\mathbf{r}(t)+\frac{D^{\circ}}{k T} \mathbf{F}(t) \delta t+\mathbf{R},
$$

where $T$ is the temperature, $k$ is the Boltzmann constant, $D^{\circ}$ is the self-diffusion coefficient of the particles at infinite dilution, $\delta t$ is the time increment, $\mathbf{r}$ is the $3 N$-dimensional configuration vector, and $\mathbf{F}$ is the total force acting on the particles at the beginning of the step. $\mathbf{R}$ is a random displacement, chosen from a Gaussian distribution with zero mean, $\langle\mathbf{R}\rangle=\mathbf{0}$, and variance $\left\langle\mathbf{R R}^{T}\right\rangle=2 D^{\circ} \delta t \mathbf{I}$, where $\mathbf{I}$ is the identity matrix. Since the simulation box contains charged particles, we improve the efficiency of the simulation by evaluating the probability of each displacement with a smart Monte Carlo criterion [42, 43].

Solvent-averaged interactions between ions are assumed to be pairwise additive, and are computed using the following interaction potential, which contains pairwise repulsive interactions and the Coulomb part:

$$
V_{i j}(r)=\frac{1}{4 \pi \varepsilon_{0} \varepsilon_{r}}\left[\frac{\left|q_{i} q_{j}\right|}{12\left(a_{i}+a_{j}\right)}\left(\frac{a_{i}+a_{j}}{r}\right)^{12}+\frac{q_{i} q_{j}}{r}\right]
$$

Here $a_{i}$ is the radius of the $i$ th ion, $\varepsilon_{0}$ the dielectric constant of the vacuum, $\varepsilon_{r}$ the relative dielectric constant of the pure solvent, taken equal to 78.54. The minimum of the cation-anion potential corresponds to the sum of the radii $a_{i}+a_{j}$. The ionic radii used in this paper are $1.68 \AA$ for the sodium ion and $1.99 \AA$ for the chloride ion. These parameters correspond to hydrated radii, and have already proved to describe accurately structural
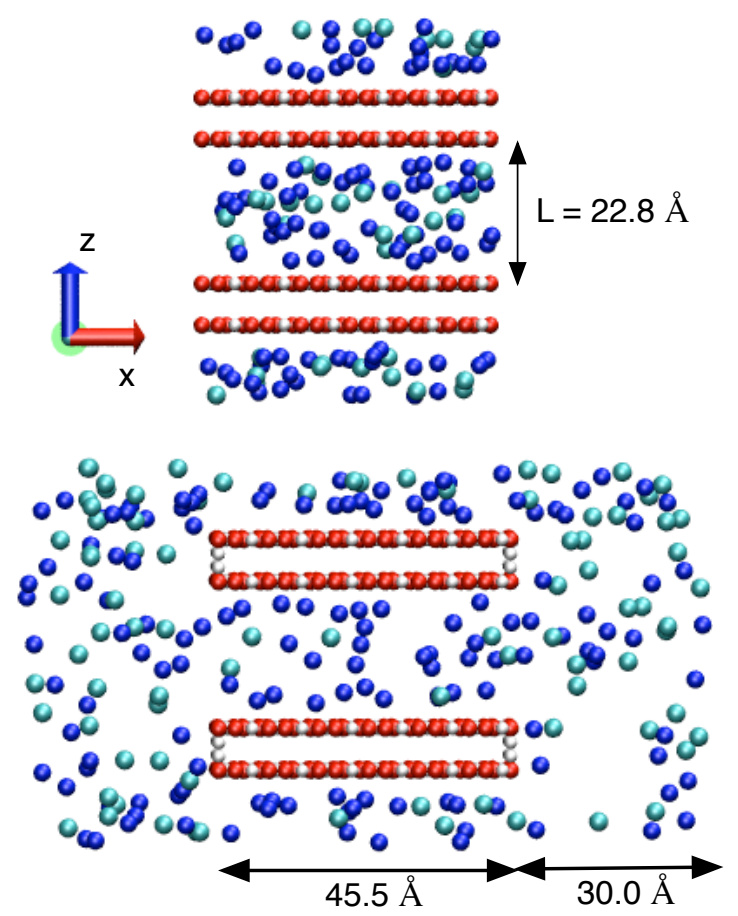

FIG. 4: Snapshots of the simulation boxes of Brownian Dynamics. Sodium ions are in blue and chloride ions are in cyan. The clay layers are infinite in the $y$-direction. Top: Simulation box used to fit the coarse-grained repulsion parameter between ions and the clay surface, which contains infinite clay layers. Bottom: Simulation box used to study the Donnan equilibrium. The length of the reservoir in the $x$-direction is $60 \AA$.

and transport properties of ions in bulk electrolyte solutions $[36,44,45]$.

The interaction potential between ions and the neutral and charged spheres representing the clay layer is also assumed to be given by eq. 3. Note that the repulsive contribution between a neutral sphere of the clay and an ion is supposed to be exactly the same as the one between a charged sphere of the clay and an ion. The short-range repulsion between ions and clays is then controlled by the parameter $a_{i}$ relative to the spheres of the clay. The fitting procedure of this parameter, denoted $a$ in the following, is presented below (see Sec. III A).

In every case, the simulation box contains two clay layers with an interlayer distance $L$ which is the distance between the centers of the spheres representing the clay surface. The width of one clay layer is equal to $6.54 \AA$ (z-direction) and its horizontal dimensions are $45.5 \times 45.0 \AA^{2}$. The net negative charge of these clay surfaces is equal to $60 e$, with $e$ the elementary charge. In the following, $L$ takes the values $12.8,17.8,22.8$ or $32.8 \AA$. The interlayer space is filled with 60 sodium counterions. Two different cases are studied in what follows. In the first one, the simulation box also contains sodium chloride at a molar concentration $C_{\text {salt }}$. Owing to the periodic boundary conditions in all dimensions, this corresponds 
to a periodic stacking of infinite clay layers is simulated. This simulation box is used to fit the coarse-grained repulsion parameter between ions and the clay (see Sec. III A). In the second case, the finite clay layers and their counterions are in contact with a reservoir which contains an electrolyte solution. Again, the simulation box is used with periodic boundary conditions in the three directions. Fig. 4 shows both types of simulation boxes for an interlayer distance $L$ equal to $22.8 \AA$. The dimension of the boxes in the direction $y$ parallel to the clay surfaces is always equal to $45 \AA$.

To compute the soft core interactions we use a spherical cutoff of half a box length, applying the minimum image convention. Coulomb interactions are computed using three dimensional Ewald summation with the conducting boundary condition.

\section{Density Functional Theory}

\section{Free energy functional}

We also calculated ion properties at the coarse-grained level of description (averaged over solvent configurations) from Density Functional Theory (DFT) [46, 47]. The system then reduces to a three-component "mixture" consisting of (i) colloidal layers assumed to be parallel and immobile (ii) counterions of charge $+\nu e$; and (iii) coions of charge $-\nu e$. A semi-grand-canonical description is adopted whereby the system is in osmotic equilibrium with an ionic solution containing a symmetric salt made up of the same point co- and counterions; this fixes the chemical potential of the salt, $\mu_{s}=\mu_{+}+\mu_{-}$which is the sum of the cationic and anionic ones.

The fundamental quantities in DFT are the equilibrium density profiles $\rho_{+}(\mathbf{r})$ and $\rho_{-}(\mathbf{r})$ of counterions and coions in the "external" field of the clay layers. According to the basic principles of DFT [48] they may be determined by minimizing the grand potential functional

$\Omega\left[\rho_{+}(\mathbf{r}), \rho_{-}(\mathbf{r})\right]=\mathcal{F}\left[\rho_{+}(\mathbf{r}), \rho_{-}(\mathbf{r})\right]-\sum_{\alpha=+,-} \mu_{\alpha} \int_{V} \rho_{\alpha}(\mathbf{r}) \mathrm{d} \mathbf{r}$.

$\mathcal{F}$ is the free energy functional, which is conventionally split into ideal, external, Coulombic, and correlational contributions [49]:

$$
\mathcal{F}=\mathcal{F}\left[\rho_{+}(\mathbf{r}), \rho_{-}(\mathbf{r})\right]=\mathcal{F}_{\text {id }}+\mathcal{F}_{\text {ext }}+\mathcal{F}_{\text {Coul }}+\mathcal{F}_{\text {corr }}
$$

The bulk concentrations differ from those in the reservoir for any given chemical potential $\mu_{s}=\mu_{+}+\mu_{-}$. The various contributions to the functional (5) are

$$
\mathcal{F}_{\mathrm{id}}=k_{\mathrm{B}} T \sum_{\alpha=+,-} \int_{V} \rho_{\alpha}(\mathbf{r})\left(\ln \left(\rho_{\alpha}(\mathbf{r}) \Lambda_{\alpha}^{3}\right)-1\right) \mathrm{d} \mathbf{r},
$$

where $\Lambda_{\alpha}$ is the de Broglie thermal wavelength of ion species $\alpha$. The external field contribution may be cast in the form

$$
\mathcal{F}_{\text {ext }}=\sum_{\alpha=+,-} \int_{V} \rho_{\alpha}(\mathbf{r}) U_{\alpha}(\mathbf{r}) \mathrm{d} \mathbf{r} .
$$

The (McMillan-Mayer) external potentials due to the interactions with the clay surfaces $U_{\alpha}(\mathbf{r})$ are supposed to be homogeneous. The atomic structure of the clay is ignored. They are modelled by a hard wall with a uniform charge density equal to $\sigma=-0.00805 \times e$ per $\AA^{2}$, equivalent to the charge density at the atomic level. The minimal approach distance between ions and the clay surfaces is obtained from MD simulations thanks to a coarse-graining procedure (see Sec. III A).

The mean-field Coulomb contribution is of the familiar form

$$
\mathcal{F}_{\text {Coul }}=\frac{\nu^{2} e^{2}}{8 \pi \epsilon_{0} \epsilon_{r}} \int_{V} \mathrm{~d} \mathbf{r} \int_{V} \mathrm{~d} \mathbf{r}^{\prime} \frac{\rho(\mathbf{r}) \rho\left(\mathbf{r}^{\prime}\right)}{\left|\mathbf{r}-\mathbf{r}^{\prime}\right|}
$$

where $\rho(\mathbf{r})=\rho_{+}(\mathbf{r})-\rho_{-}(\mathbf{r})$ is the charge density of the microions (divided by $\nu e$ ). No exact expression is known for the correlation term, for which we adopt the local density approximation (LDA):

$$
\mathcal{F}_{\text {corr }}=\int_{V} F_{\text {exc }}^{0}\left(\rho_{+}(\mathbf{r}), \rho_{-}(\mathbf{r})\right) \mathrm{d} \mathbf{r}
$$

where $F_{\text {exc }}^{0}$ denotes the excess free energy per unit volume of a uniform system of $\rho_{-}$and $\rho_{+}$co- and counterions per unit volume, in a neutralizing uniform background, representing the smeared-out charge of the clay layer. Simple mean-field approaches, such as the PoissonBoltzmann theory, simply neglect this correlation term. For concentrated electrolyte solutions, this approximation does not hold anymore: ionic correlations become important, especially at molar concentration for which excluded volume effects perturb the electrostatic force. We treat the correlation effects within the Mean Spherical Approximation (MSA) [50]. This theory is based on the primitive model of electrolytes where ions are modeled by charged hard spheres. The MSA generalizes the Debye-Huckel theory of electrolytes by accounting for the excluded volume of the ions in the ionic atmospheres. For bulk solutions, it can be applied to model simple salts up to molar concentrations.

A difficulty arises from the fact that for bulk electrolyte solutions the derivation of the MSA $[51,52]$ is obtained for a neutral ionic system $\rho_{+}=\rho_{-}$whereas in our case the electro-neutrality condition is only satisfied thanks to a uniform background. In fact, the solution of the MSA in that case $[53,54]$ is similar to the case for which a background is not necessary, except for a trivial renormalization of the charge carried by the spheres. If we consider an excluded neutralizing background [55], this charge renormalization can be omitted. The only parameter of this MSA model is the diameter of the ions, supposed to be the same for cations and anions. The MSA diameter used in this paper is $3.3 \AA$. This diameter 
is the average of the diameters of $\mathrm{Na}^{+}$and $\mathrm{Cl}^{-}$used in bulk MSA theory $[34,56]$, which is able to reproduce the ion properties up to molar concentrations. Moreover, it has already been shown that the MSA diameters and the one used in BD with a soft-core repulsion yield similar results $[36,57]$.

\section{Ionic density profiles}

Functional minimization of the form of the grand potential (4) with respect to the profiles $\rho_{+}(\mathbf{r})$ and $\rho_{-}(\mathbf{r})$ :

$$
\frac{\delta \Omega\left[\rho_{+}(\mathbf{r}), \rho_{-}(\mathbf{r})\right]}{\delta \rho_{\alpha}(\mathbf{r})}=\frac{\delta \mathcal{F}}{\delta \rho_{\alpha}(\mathbf{r})}-\mu_{\alpha}=0 ;
$$

leads to the following coupled Euler-Lagrange equations for the unknown profiles:

$$
\begin{gathered}
k_{\mathrm{B}} T \ln \left(\rho_{\alpha}(\mathbf{r}) \Lambda_{\alpha}^{3}\right)+U_{\alpha}(\mathbf{r}) \\
+\alpha \int \frac{\nu^{2} e^{2}}{4 \pi \epsilon_{0} \epsilon_{r}} \frac{\rho_{+}\left(\mathbf{r}^{\prime}\right)-\rho_{-}\left(\mathbf{r}^{\prime}\right)}{\left|\mathbf{r}-\mathbf{r}^{\prime}\right|} \mathrm{d} \mathbf{r}^{\prime} \\
+\mu_{\alpha}^{\operatorname{exc}}\left(\rho_{+}(\mathbf{r}), \rho_{-}(\mathbf{r})\right)-\mu_{\alpha}=0
\end{gathered}
$$

with $\alpha=+,-$. The excess chemical potential of ion $\alpha$, $\mu_{\alpha}^{\text {exc }}\left(\rho_{+}(\mathbf{r}), \rho_{-}(\mathbf{r})\right)$ is calculated from the MSA.

If $\mathcal{F}_{\text {corr }}$ is set to zero, $\mu_{\alpha}^{\text {exc }}\left(\rho_{+}(\mathbf{r}), \rho_{-}(\mathbf{r})\right)=0$ and minimization of the grand potential functional (4) is equivalent to solving the Poisson-Boltzmann (PB) equation [48]. In the general case of lamellar system, there is no analytical solution of the $\mathrm{PB}$ equation, the classical Gouy-Chapman being valid only for a single interface. Nevertheless it is easily solved numerically. If correlations are taken into account at the MSA level of approximation, the numerical solution can be found by a similar method. We solved the two DFTs (PB and MSA) for the various hydration states. The $\mathrm{PB}$ completely neglects the correlations between the ions, but it is still the basic theory for the mesoscopic description of hydrated clays so that it is important to check its domain of validity. The MSA functional takes into account the correlations between the ions, both for the electrostatic force and the hard sphere repulsion, but it is a local functional.

\section{RESULTS}

\section{A. Coarse-graining of the ion/surface repulsion}

As stated before, in Brownian Dynamics (BD) simulations, the ion/clay interaction potential is assumed to contain a short-range repulsion whose range is controlled by the parameter $a$, proportional to a distance (see. Eq. 3 ). This coarse-grained parameter $a$ is obtained from the calculation of the density profiles of co-ions and counterions between the clay surfaces, in the $z$-direction perpendicular to the clay surface. The density profiles are computed from Molecular Dynamics (MD) simulations

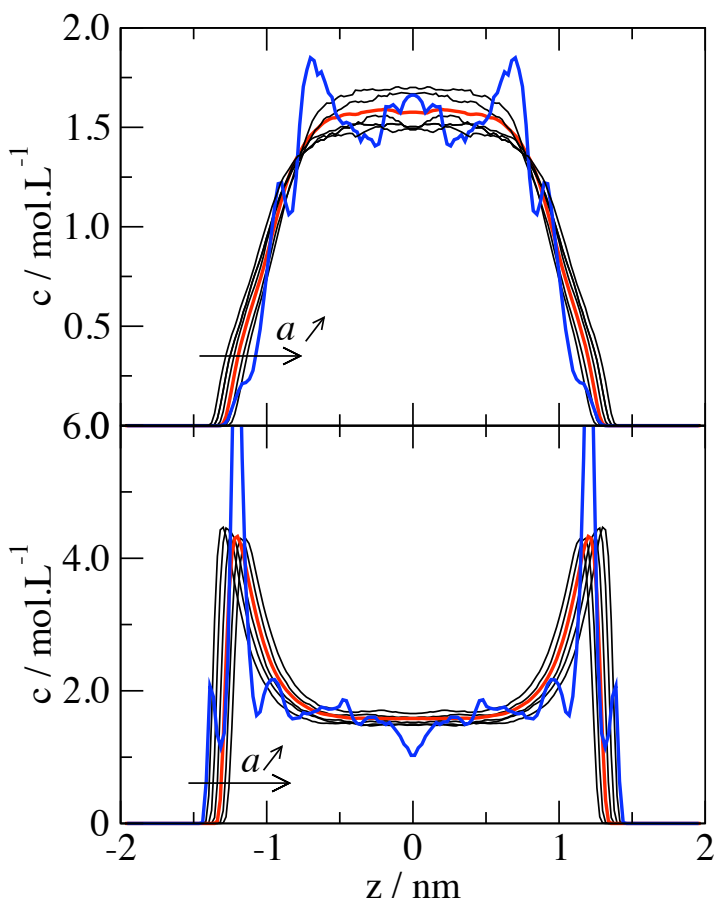

FIG. 5: Density profiles of ions in the direction perpendicular to the clay layers for an interlayer distance $L=32.8 \AA$ and a concentration of added electrolyte $C_{\text {salt }}=0.96$ mol. $\mathrm{L}^{-1}$, in a system of infinite clay layers. Molecular dynamics calculations in blue and Brownian Dynamics calculations in black, for several values of parameter $a$, between 2.6 and $4.6 \AA$, and in red, for the value $a=3.8 \AA$. Top: Density profiles of coions (chloride ions), Bottom: Density profiles of counterions (sodium ions).

in a system of infinite clay layers with a large interlayer spacing $(L=32.8 \AA)$ and a concentration of added electrolyte solution equal to 0.96 mol. $\mathrm{L}^{-1}$. More precisely, the MD simulation box contains 1454 water molecules, 28 chloride ions and 52 sodium ions. The density profiles of ions are also computed from Brownian Dynamics at the coarse-grained level, for several values of the repulsion parameter $a$, ranging from 2.6 to $4.6 \AA$ by steps of $0.4 \AA$. The mesoscopic BD simulation box also contains an electrolyte at a concentration equal to $0.96 \mathrm{~mol}^{-1} \mathrm{~L}^{-1}$ for an interlayer spacing of $32.8 \AA$, which corresponds to 1440 neutral and charged immobile spheres to describe the infinite clay layers, 140 sodium ions and 80 chloride ions to describe the confined electrolyte. The density profiles obtained from MD and from BD with several repulsion parameters $a$ are shown in Fig. 5. From the plots shown in this figure, we deduce that the best value for the repulsion parameter is $a=3.8 \AA$. For this repulsion parameter, the position of the peak of the counterions density profile is the same in $\mathrm{BD}$ as in $\mathrm{MD}$, and the coions density profile is non-zero in $\mathrm{BD}$ at the same distance from the clay than in MD (see Fig. 6-top).

As can be seen in Fig. 6-bottom, the integrals of 


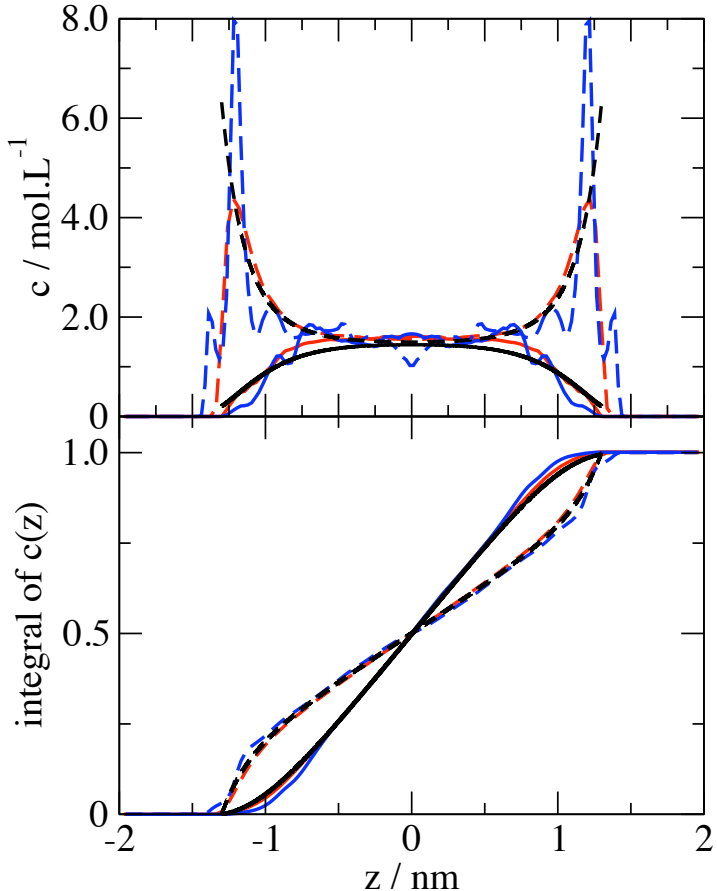

FIG. 6: Density profiles of ions in the direction perpendicular to the clay surface (top) and their integrals (bottom) for an interlayer spacing $L=32.8 \AA$ and a concentration of added electrolyte $C_{\text {salt }}=0.96 \mathrm{~mol} . \mathrm{L}^{-1}$ in a system of infinite clay layers. The density profiles of counterions are in dashed lines and the ones of coions in plain lines. Molecular dynamics calculations in blue, Brownian Dynamics calculations in red for the parameter $a=3.8 \AA$, DFT/MSA calculations in black.

the density profiles as funtions of the distance $z$ to the clay surfaces obtained from $\mathrm{MD}$ or from $\mathrm{BD}$ with $a=3.8 \AA$ are also in good agreement. Fig. 6 also gives the density profiles and their integrals obtained from DFT/MSA calculations. For this last method, again, a single parameter, namely the distance of minimal approach of the ions from the clay, is adjusted. The best results are obtained when it is equal to the position of the oxygen atoms of the clay surface increased by $3.4 \AA$. As shown in Fig. 6, with this parameter the DFT/MSA density profiles and their integrals are in good agreement with those obtained from MD and BD. It should be noted that the coarse-grained ion/clay repulsion adjusted from $\mathrm{BD}$ and DFT/MSA are actually similar. On the one hand, within the DFT/MSA calculation, the minimal distance of approach corresponds to hard-cores and it is equal to $3.4 \AA$. On the other hand, in BD simulations, the repulsion corresponds to a soft-core one, and the parameter is $3.8 \AA$. The BD parameter is larger than the DFT/MSA one, because this soft repulsion allows the ions to come closer to the surface than the distance corresponding to the minimum of the interaction potential. Moreover, it has been shown previously [39] that the sodium ion is hydrated in the vicinity of the clay surface

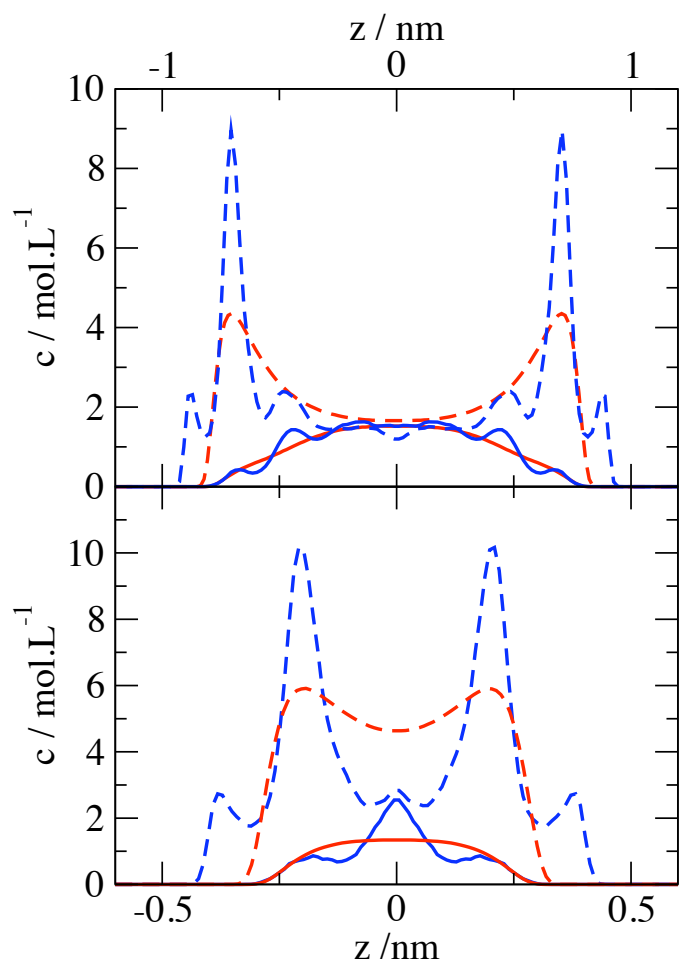

FIG. 7: Density profiles of ions in the direction perpendicular to the clay surfaces in a system of infinite clay layers. Top: Interlayer space $L=22.8 \AA$, concentration of added electrolyte $C_{\text {salt }}=0.73$ mol.L ${ }^{-1}$; Bottom: Interlayer space $L=12.8 \AA$, concentration of added electrolyte $C_{\text {salt }}=0.43$ mol.L $\mathrm{L}^{-1}$. The density profiles of counterions are in dashed lines and the ones of coions in plain lines. Molecular dynamics calculations in blue, Brownian Dynamics calculations in red.

so that this rather large distance of minimal approach to the surface is not surprising.

The ion/clay repulsion parameter is assumed to be independent on the interlayer distance, since it accounts for the non-electrostatic short-range interactions between ions and the clay surface. We checked that the coarsegrained repulsion used in $\mathrm{BD}$ simulations allows us also to capture the essential features of the system for other interlayer distances and other concentrations. Fig. 7top shows the density profiles of ions for an interlayer distance $L=22.8 \AA$ and a concentration of added electrolyte equal to $C_{\text {salt }}=0.73$ mol. $\mathrm{L}^{-1}$. Again, the peak of the counterion density profile obtained from $\mathrm{BD}$ has the same position as in MD and the BD density profile of coions is non-zero at the same distance as in MD. Of course, the density profiles at the coarse-grained level do not present any of the oscillations observed in Molecular Dynamics since those oscillations are mainly due to the layering of water molecules $[58,59]$. The same is observed for the smallest interlayer distance $(L=12.8 \AA$, see Fig. 7-bottom). In this case, the influence of water molecules on the ionic distribution is high since only about three 


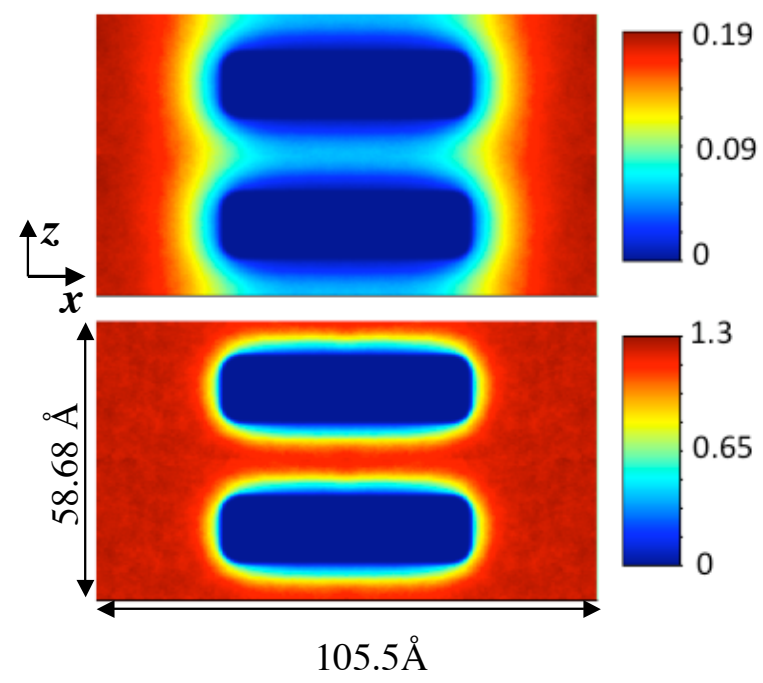

FIG. 8: Average distribution functions of coions in the simulation box for an interlayer distance $L=22.8 \AA$. Top: $C_{\text {ext }}=0.18$ mol. $\mathrm{L}^{-1}$. Bottom: $C_{\text {ext }}=1.23 \mathrm{~mol} . \mathrm{L}^{-1}$. Note that to increase the statistical accuracy, these distribution functions have been computed only for positive values of $x$ and $z$ (i.e. for a quarter of the simulation box).

layers of water molecules are present between the clay layers.

\section{B. Equilibrium with a reservoir}

To study the insertion of ions in the micropore from BD simulations, the following procedure is used. First, counterions and coions are placed randomly in the reservoir of the simulation box, at an initial external concen-

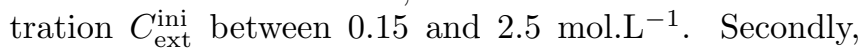
this system is equilibrated during $10^{6}$ to $6 \times 10^{6}$ time steps, the number of time steps depending on the total number of moving particles (it is increased when the total number of particles is decreased). The value of the time step also depends on the system and varies between $0.03 \times 10^{-12}$ and $0.2 \times 10^{-12} \mathrm{~s}$. Once the system is equilibrated, the distribution functions of ions are averaged over 20 runs of about $10^{6}$ time steps each (again, the number of time steps per run depends on the total number of mobile particles in the simulation box). More precisely, the local ionic concentration as a function of the position $(x, z)$ in the simulation box is computed on a grid of $200 \times 200$ points (only for positive values of $x$ and $z$, taking advantage of the symmetry of the simulation box). From these distribution functions, the mean ion concentration in the reservoir $\left(C_{\text {ext }}\right)$ and in the interlayer space $C_{\text {int }}$ at equilibrium are computed. As for the external concentration, this quantity is obtained by averaging the distribution functions over every $z$ value but only for $|x|>100.5 \AA$, i.e. over a slab of $10 \AA$ width far from the clay surfaces. The internal concentration is obtained

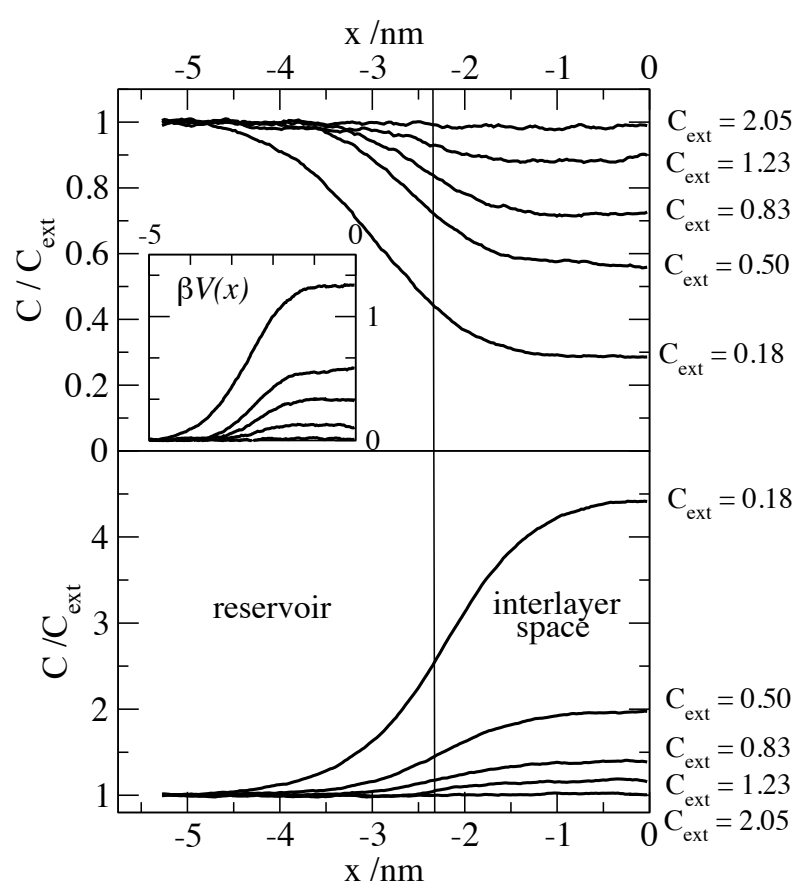

FIG. 9: Ionic concentration as a function of the distance $x$ parallel to the clay surface obtained from Brownian Dynamics simulations (this function is given for half a simulation box), divided by the ionic concentration in the reservoir (denoted by $\left.C_{\text {ext }}\right)$. The electrolyte concentration in the reservoir is indicated in the side of the figure, in mol. $\mathrm{L}^{-1}$. Top: Concentration of coions (namely chloride ions). The inserted plot represents the potential of mean force on a single ion as a function of $x$. Bottom: Concentration of counterions (namely sodium ions). The clay layer is situated between $x=-2.34 \mathrm{~nm}$ and $x=0 \mathrm{~nm}$.

by averaging the distribution functions over all $z$ values but only for $|x|<5 \AA$, i.e. over a slab of $10 \AA$ width perpendicular to the clay surface in the middle of the micropore (whose total length along the $x$-direction is $46.8 \AA$ ). This implies that the volume used to compute the internal concentration always includes the volume of the clay layers. We checked for two of the systems that different initial configurations of the simulation box lead to the same equilibrium states, i.e. to the same values of $C_{\text {int }}$ and $C_{\text {ext }}$ (all ions are placed either in the micropore, or in the reservoir, or everywhere except inside the clay).

Typical distribution functions of coions obtained from BD for an interlayer distance $L=22.8 \AA$ are displayed in Fig. 8. These distribution functions correspond to external electrolyte concentrations at the equilibrium equal to 0.18 mol.L $\mathrm{L}^{-1}$ (Fig. 8-Top) and to $1.23 \mathrm{mol.L}^{-1}$ (Fig. 8Bottom). This figure shows that the insertion of coions in the micropore space increases strongly when the external concentration is increased, as expected. To have more insight into this question, we have also plotted the "local" co- and counterions concentrations (i.e. averaged over a thin layer of $4 \AA$ width parallel to the clay surfaces and in the middle of the interlayer space) as functions of the 
abcissa $x$, for different external concentrations (see Fig. 9). To enable a comparison between different cases, these concentrations are renormalized by the external concentration. As expected, the insertion of coions increases significantly as the external concentration is increased: anions can enter the interlayer space provided that the charged surfaces are screened by the Debye layer, i.e. $\kappa L>1$ with $\kappa$ the inverse of the Debye length and $L$ the interlayer distance, as observed in other studies $[3,60]$. Moreover, these plots allow us to evaluate the energy barrier which must be overcome by the coions to penetrate into the interlayer space:

$$
V(x)=-k_{\mathrm{B}} T \ln \left(C(x) / C_{\text {ext }}\right)
$$

where $V$ is the potential of mean force on a single anion for abcissa $x$. As the external concentration goes from 0.18 to 2 mol.L ${ }^{-1}$, this barrier decreases from about $1 k_{\mathrm{B}} T$ to zero. (see the insert in Fig. 9). Fig. 9 also shows that the edge effect due to the finite size of the clay layers depends on the salt concentration. At small $\kappa$ values (i.e. at small concentrations), the screening length is large so that the influence of the clay ranges over wide distances in the reservoir. Interestingly, for the coions, the edge domain is mainly in the reservoir part whereas for the counterions it is mainly in the interlayer space.

Fig. 10 displays the ratio between the internal concentration of coions and their external concentration, as a function of the external concentration. Note that in this plot, the internal concentration is not a local value in the interlayer space, but the number of coions divided by the whole volume of the porous medium, including the volume of the clay layers. Thus, the ratio $C_{\text {int }} / C_{\text {ext }}$ tends to 1 only if the volume occupied by the clays becomes negligible with respect to the interlayer spacing, i.e. the interlayer spacing becomes very large. Several results are compared on Fig. 10: for four different interlayer distances, the results of Brownian Dynamics simulations are compared to DFTs calculations, either in the PoissonBoltmann treatment or in the MSA. The diameter of circles used to represent BD results in Fig. 10 corresponds to the uncertainty of the result, estimated from the standard deviation of different runs. The shape of the curves as a function of $C_{\text {ext }}$ is the same with all methods: As expected, the internal concentration of coions tends to 0 when $C_{\text {ext }}$ tends to 0 and is a growing function of $C_{\text {ext }}$. Fig. 10 also shows that DFT/MSA calculations yield a good agreement with BD calculations for the two highest interlayer distances (namely for $L \geq 22.8 \AA$ ). At small interlayer distances, the DFT result, which is based on a local density approximation, does not account for the strong correlation effects between ions. Thus, it overestimates the insertion rate of coions.

It is worth noting that there are two regimes in the Donnan equilibrium. On the one hand, when $C_{\text {ext }}$ tends to 0 , the $\mathrm{PB}$ calculation, which treats ions as point charges, slightly underestimates the insertion rate of coions with respect to the DFT/MSA and BD results. This is due to the fact that for these small values of the

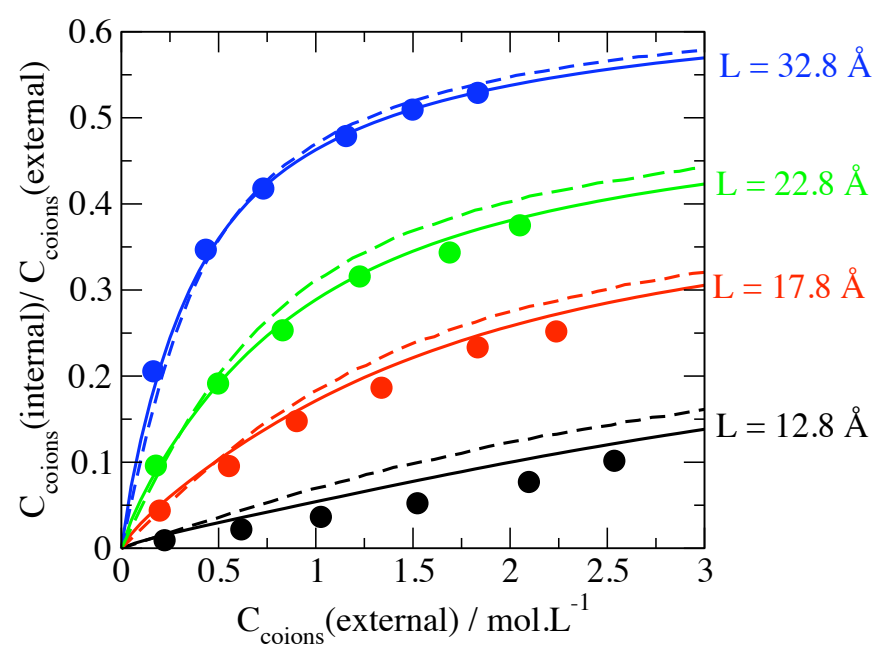

FIG. 10: Ratio between the internal concentration of coions and their external concentration as a function of the external concentration (concentration in the reservoir), for several interlayer distances. Circles: Brownian Dynamics simulation, dashed lines: Poisson-Boltzmann calculations and plain lines: DFT/MSA calulations.

electrolyte concentration, the main interactions between ions are due to electrostatics and the attraction between ions of opposite charges tends to increase the insertion of coions; in this regime, the activity coefficients of ions are smaller than 1 whereas PB calculations assume that they are equal to 1 . On the other hand, for higher external concentrations, hence for higher internal concentrations, the excluded volumes of ions becomes important, especially in the micropore. This excluded volume effect tends to prevent them from entering into the interlayer space; as the excluded volumes are not taken into account in the $\mathrm{PB}$ calculation, $\mathrm{PB}$ overestimates the insertion rate.

The influence of the coarse-grained repulsion parameter $a$ on the insertion rate of coions in the micropore is shown in Fig. 11. We performed Brownian Dynamics simulations have been performed for the interlayer distance $L=22.8 \AA$ and for three intermediate external concentrations with a repulsion parameter slightly smaller (3.0) and slightly larger (4.6) than the optimal choice based on the density profiles $(a=3.8 \AA)$. The influence of this parameter on the insertion rate is non negligible. As expected, the insertion rate increases when the repulsion parameter decreases. This underlines a posteriori the importance of a careful coarse-graining procedure to obtain accurate results from mesoscopic descriptions of this system. It should be noted that the parameter $a$ would probably be different in the presence of other type of counterions. For example, the cesium ion is known to come closer to the clay surface than the sodium ion [39]. Furthermore, we also investigate the influence of the size of the reservoir in the BD simulation box. It is shown in Fig. 11 that even with a roughly twice smaller pore 


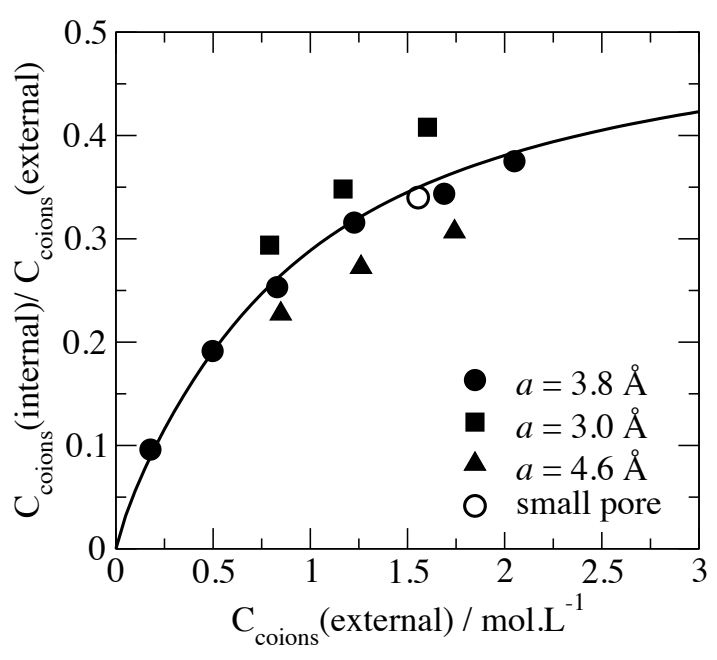

FIG. 11: Same as Fig. 10 but only for the interlayer distance $L=22.8 \AA$, for different values of the ion/clay repulsion parameter $a$. The open circle corresponds to a simulation box with a smaller pore size to represent the reservoir $(39.5 \AA$ instead of $60.0 \AA$ ).

length, we are able to describe quantitatively the Donnan equilibrium.

The effect of ionic correlations is illustrated in Fig. 12. It represents the product of the cationic and anionic concentrations in the interlayer space as a function of the external electrolyte concentrations. The function $y=x^{2}$ is also plotted. In the ideal case ( $\mathrm{PB}$ equation, activity coefficients of all ions equal to 1 ), the equality of the chemical potentials of the electrolyte inside and outside the interlayer space yields $\left(C_{+} C_{-}\right)_{\text {int }}=\left(C_{+} C_{-}\right)$ext where $C_{+}$and $C_{-}$are the concentrations of cations and anions, respectively. Thus, if this equality is verified, we expect a data collapse on the parabolic curve $y=x^{2}$. It is shown in Fig. 12 that this equality is roughly verified even if the activity coefficients of ions are different from 1. Nevertheless, there are small departures from the ideal case. They can be understood by taking into account the activity corrections, because

$$
\frac{\left(C_{+} C_{-}\right)_{\mathrm{int}}}{\left(C_{+} C_{-}\right)_{\mathrm{ext}}}=\frac{\left(\gamma_{+} \gamma_{-}\right)_{\mathrm{ext}}}{\left(\gamma_{+} \gamma_{-}\right)_{\mathrm{int}}}
$$

where $\gamma_{+}$(resp. $\gamma_{-}$) is the activity coefficient of the cation (resp. anion). For large interlayer distances and large external concentrations, both in the interlayer space and in the reservoir, the activity coefficients of ions are increasing functions of the concentration (a repulsive regime is observed). As the ionic concentration is larger in the reservoir than in the interlayer space, in this case the ratio $\left(\gamma_{+} \gamma_{-}\right)_{\text {ext }} /\left(\gamma_{+} \gamma_{-}\right)_{\text {int }}$ is larger than one, thus the product $\left(C_{+} C_{-}\right)_{\text {int }}$ is larger than $\left(C_{+} C_{-}\right)_{\text {ext }}$. For small interlayer distances, the excess of counterions that neutralize the clay medium in the interlayer space increases the internal concentration so that the activity coefficients are larger inside the pore than outside. In

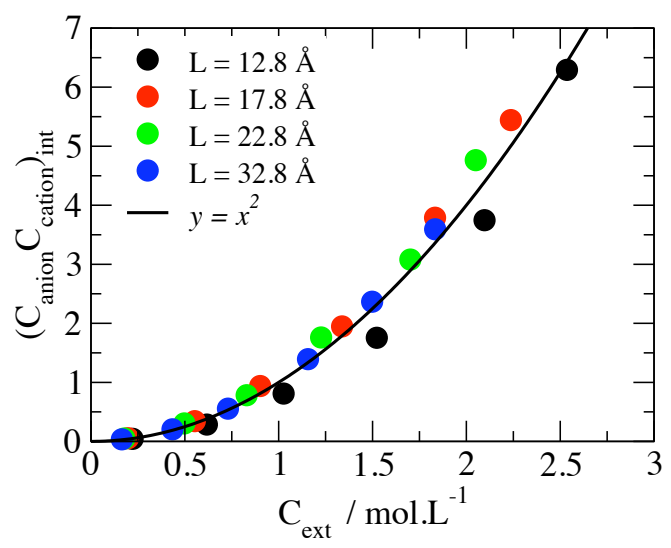

FIG. 12: Product of the local concentrations of coions and counterions in the interlayer space obtained from Brownian Dynamics as a function of the electrolyte concentration in the reservoir.

this case, $\left(C_{+} C_{-}\right)_{\text {int }}$ is thus smaller than its ideal value $\left(C_{+} C_{-}\right)_{\text {ext }}$.

\section{CONCLUSION}

We developed a coarse-graining strategy to study the exclusion properties of salt in Montmorillonite clays, which is an example of lamellar porous medium. We first calibrate a mesosopic description of the system using Molecular Dynamics simulation at the atomic scale, and then use it to derive the salt-exclusion properties in a variety of porosity and salinity conditions The first coarse-graining step is to treat the solvent as a continuous medium and to adopt a simplified but realistic description of the clay layers. Then, the two-body correlations (between ions) are assumed to be the same as in bulk electrolyte solutions. For the one-body correlations (between ions and the clay surface), we use the continuous model for electrostatics and a repulsive potential. The latter is adjusted from results obtained at the atomic scale by Molecular Dynamics simulations. The value of this repulsion parameter is found to have a crucial influence on the Donnan effect. If other types of counterions, with other hydration properties (in particular in the vicinity of the clay surface) are studied, the coarse-graining procedure will have to be repeated.

We have shown that the salt exclusion increases when $\kappa L$ decreases (where $\kappa$ is the inverse of the Debye length and $L$ the interlayer distance) and that this effect is modulated by the correlations between ions. We have identified two different regimes. At low concentration in the reservoir, we observe a regime controlled by electrostatics: the Coulomb attraction between ions increases the amount af salt in the interlayer space. On the opposite, at high concentration in the reservoir, the excluded volume effect dominates. This repulsion strongly reduces the amount of salt entering in the interlayer space, es- 
pecially for low hydration states of the clay (i.e. small interlayer distances). Of course, when only one or two layers of water molecules are present in the interlayer space, the resort to atomistic simulations is required to study structural and dynamical properties of ions in these porous media [30]. But, as far as very hydrated clays are under study, theoretical investigations must be carried at a mesoscopic scale. We show in this paper how the mesoscopic model may be calibrated on the atomic one. Moreover, the interest of Brownian Dynamics simulations is that they do not only provide structural results but also enable to investigate dynamical properties of ions.
In a future work, we will study the diffusion properties of ions in the interlayer space in every direction using BD. Another interesting question concern the so-called diffusiophoresis effect, which is related to the possible motion of clay layers in the presence of a gradient of electrolyte concentration [61]. We aim in the near future at studying this effect which is the dynamical reciprocal of the motion of ions with respect to the clay surface.

Acknowledgments: One of the authors (B. R.) acknowledges financial support from the Agence Nationale pour la gestion des Déchets RadioActifs (ANDRA).
[1] ANDRA, Évaluation de la faisabilité du stockage géologique en formation argileuse, Dossier 2005 Argile : Synthèse, Châtenay-Malabry, France, 2005.

[2] M. Bradbury and B. Baeyens, Near Field Sorption Data Bases for Compacted MX-80 Bentonite for Performance Assessment of a High-Level Radioactive Waste Repository in Opalinus Clay Host Rock, Paul Scherrer Institut, Switzerland, 2003.

[3] M. Dubois, T. Zemb, L. Belloni, A. Delville, P. Levitz, and R. Setton, J. Chem. Phys. 96, 2278 (1992).

[4] R. Qiao and N. Aluru, J. Chem. Phys. 118, 4692 (2003).

[5] R. QIAO and N. Aluru, International Journal for Multiscale Computational Engineering 188, 173 (2004).

[6] S. Joseph and N. Aluru, Langmuir 22, 9041 (2006).

[7] D. Horinek and R. Netz, Phys. Rev. Lett. 99, 226104 (2007).

[8] N. Skipper, K. Refson, and J. MCConnell, Clay Minerals 24, 411 (1989).

[9] F.-R. Chang, N. Skipper, and G. Sposito, Langmuir 11, 2734 (1995)

[10] F.-R. Chang, N. Skipper, and G. Sposito, Langmuir 13, 2074 (1997).

[11] D. Smith, Langmuir 14, 5959 (1998).

[12] G. Sposito, N. T. Skipper, R. Sutton, S. H. Park, A. K. Soper, and J. A. Greathouse, Proc. Nat. Acad. Sci. 96, 3358 (1999).

[13] E. Ferrage, B. Lanson, N. Malikova, A. Plancon, B. Sakharov, and V. Drits, Chem. Mater. 17, 3499 (2005).

[14] E. Boek, P. Coveney, and N. Skipper, J. Am. Chem. Soc. 117, 12608 (1995).

[15] D. Young and D. Smith, J. Phys. Chem. B 104, 9163 (2000).

[16] H. Whitley and D. Smith, J. Chem. Phys. 120, 5387 (2004).

[17] X.-D. Liu and X.-C. Lu, Angew. Chem. Int. Ed. 45, 6300 (2006).

[18] E. Hensen, T. Tambach, A. Bliek, and B. Smit, J. Chem. Phys. 115, 3322 (2001).

[19] T. Tambach, P. Bolnuis, and B. Smit, Angew. Chem. Int. Ed. 43, 2650 (2004).

[20] T. Tambach, P. Bolhuis, E. Hensen, and B. Smit, Langmuir 22, 1223 (2006).

[21] R. Sutton and G. Sposito, J. Coll. Interf. Sci. 237,
174 (2001).

[22] V. Marry, P. Turq, T. Cartalller, and D. Levesque, J. Chem. Phys. 117, 3454 (2002).

[23] V. Marry and P. Turq, J. Phys. Chem. B 107, 1832 (2003).

[24] N. Malikova, V. Marry, J.-F. Dufrêche, C. Simon, P. Turq, and E. Giffaut, Mol. Phys. 102, 1965 (2004).

[25] N. Malikova, V. Marry, J.-F. Dufrêche, and P. Turq, Curr. Opinion in Coll. Int. Sci. 9, 124 (2004).

[26] N. Malikova, A. Cadène, V. Marry, E. Dubois, P. Turq, J.-M. Zanotti, and S. Longeville, Chem. Phys. 317, 226 (2005).

[27] N. Malikova, A. Cadène, V. Marry, E. Dubois, and P. Turq, J. Phys. Chem. B 110, 3206 (2006).

[28] R. de Carvalho and N. Skipper, J. Chem. Phys. 114, 3727 (2001).

[29] J. A. Greathouse and R. T. Cygan, Phys. Chem. Chem. Phys. 7, 3580 (2005).

[30] B. Rotenberg, V. Marry, R. Vuilleumier, N. MaLikova, C. Simon, and P. TurQ, Geochim. et Cosmochim. Acta 71, 5089 (2007).

[31] G. W. Brindley and G. Brown, Crystal Structures of Clay Minerals and their X-ray Identification, Mineralogical Society, London, 1980.

[32] E. Maegdefrau and U. Hoffman, Z. Kristallogr. Kristallgeom. Kristallphys. Kristallchem. 98, 299 (1937).

[33] J. Barthel, H. Krienke, and W. Kunz, Physical Chemistry of Electrolyte Solutions, Springer, 1998.

[34] J.-F. Dufrêche, O. Bernard, S. Durand-Vidal, and P. Turq, J. Phys. Chem. B 109, 9873 (2005).

[35] A. R. Altenberger H. L. Friedman, J. Chem. Phys. 78, $4162(1983)$

[36] J.-F. DuFrÊChe, M. Jardat, T. Olynyk, O. Bernard, and P. Turq, J. Chem. Phys. 117, 3804 (2002).

[37] H. Berendsen, J. Grigera, and T. Straatsma, J. Phys. Chem. 91, 6269 (1987).

[38] S. Koneshan, C. Rasaiah, R. Lynden-Bell, and S. LeE, J. Phys. Chem. B 102, 4193 (1998).

[39] V. Marry, B. Rotenberg, and P. Turq, Phys. Chem. Chem. Phys. 10, 4802 (2008).

[40] D. Frenkel and B. Smit, Understanding Molecular Simulations, From Algorithms to Applications, Academic Press, 2002.

[41] D. L. Ermak, J. Chem. Phys. 62, 4189 (1975).

[42] P. J. Rossky J. D. Doll H. L. Friedman, J. Chem. 
Phys. 69, 4628 (1978).

[43] M. Jardat, O. Bernard, P. Turq, and G. R. Kneller, J. Chem. Phys. 110, 7993 (1999).

[44] M. Jardat and P. Turq, Z. Phys. Chem. 218, 699 (2004).

[45] J.-F. Dufrêche, M. Jardat, P. Turq, and B. Bagchi, J. Phys. Chem. B 112, 10264 (2008).

[46] D. Henderson, editor, Fundamentals of Inhomogeneous Fluids, Dekker, 1992.

[47] R. Evans, Adv. Phys. 28, 143 (1979).

[48] J.-P. Hansen and H. Löwen, Ann. Rev. Phys. Chem. 51, 209 (2000).

[49] H. Löwen J.-P. Hansen P. A. Madden, J. Chem. Phys. 98, 3275 (1993).

[50] W. Ebeling K. Scherwinsky, Z. phys. Chemie, Leipzig 264, 1 (1983).

[51] L. Blum, Mol. Phys. 30, 1529 (1975).

[52] L. Blum J. S. Hø ye, J. Phys. Chem. 81, 1311 (1977).

[53] M. Parrinello M.P. Tosi, Chem. Phys. Lett. 64, 579
(1979).

[54] T. Alts P. Nielba B. D'Aguanno F. Forstmann, Chem. Phys. 111, 223 (1987).

[55] J.-P. Hansen, J. Phys. C Solid State Phys. 14, L151 (1981).

[56] J.-F. Dufrêche O. Bernard P. Turq, J. Chem. Phys. 116, 2085 (2002).

[57] J.-F. Dufrêche, M. Jardat, P. Turq, and B. BAgChi, J. Phys. Chem. B 112, 10264 (2008).

[58] V. Marry, J.-F. Dufrêche, M. Jardat, and P. Turq, Mol. Phys. 101, 3111 (2003).

[59] V. MARrY, J.-F. DufrÊChE, M. JARDAT, G. Mériguet, P. Turq, and Grün, Coll. Surf. A 222, 147 (2003).

[60] P. Porion, A.-M. Faugère, and A. Delville, J. Phys. Chem. B 112, 11893 (2008).

[61] B. Abecassis, C. Cottin-Bizonne, C. Ybert, A. AdJARI, and L. BOCQUET, Nature Materials 7, 785 (2008). 